\title{
Magnetic Resonance Angiography Using Fresh Blood Imaging in Oral and Maxillofacial Regions
}

\author{
Masafumi Oda, ${ }^{1}$ Tatsurou Tanaka, ${ }^{1}$ Shinji Kito, ${ }^{1}$ Manabu Habu, ${ }^{2}$ Masaaki Kodama, ${ }^{2}$ \\ Shinya Kokuryo, ${ }^{2}$ Ikuya Miyamoto, ${ }^{2}$ Daigo Yoshiga, ${ }^{2}$ Kensuke Yamauchi, ${ }^{2}$ \\ Shinnosuke Nogami, ${ }^{2}$ Nao Wakasugi-Sato, ${ }^{1}$ Shinobu Matsumoto-Takeda, ${ }^{1}$ \\ Ayataka Ishikawa, ${ }^{3}$ Ikuko Nishida, ${ }^{4}$ Katsura Saeki, ${ }^{4}$ Kazumasa Morikawa, ${ }^{4}$ \\ Kou Matsuo, ${ }^{5}$ Yuji Seta, ${ }^{5}$ Yoshihiro Yamashita, ${ }^{6}$ Kenshi Maki, ${ }^{4}$ \\ Kazuhiro Tominaga, ${ }^{2}$ and Yasuhiro Morimoto ${ }^{1,7}$ \\ ${ }^{1}$ Department of Oral Diagnostic Science, Kyushu Dental College, 2-6-1 Manazuru, Kokurakita-ku, Kitakyushu 803-8580, Japan \\ ${ }^{2}$ Department of Oral and Maxillofacial Surgery, Kyushu Dental College, 2-6-1 Manazuru, Kokurakita-ku, Kitakyushu 803-8580, Japan \\ ${ }^{3}$ Department of Pathology, Saitama Cancer Center, Saitama 362-0806, Japan \\ ${ }^{4}$ Department of Growth and Development of Function, Kyushu Dental College, 2-6-1 Manazuru, Kokurakita-ku, \\ Kitakyushu 803-8580, Japan \\ ${ }^{5}$ Department of Oral Bioscience, Kyushu Dental College, 2-6-1 Manazuru, Kokurakita-ku, Kitakyushu 803-8580, Japan \\ ${ }^{6}$ Department of Oral and Maxillofacial Surgery, Fukuoka Dental College, Fukuoka 814-0193, Japan \\ ${ }^{7}$ Center for Oral Biological Research, Kyushu Dental College, 2-6-1 Manazuru, Kokurakita-ku, Kitakyushu 803-8580, Japan
}

Correspondence should be addressed to Yasuhiro Morimoto, rad-mori@kyu-dent.ac.jp

Received 18 July 2012; Accepted 11 September 2012

Academic Editor: Toru Chikui

Copyright (c) 2012 Masafumi Oda et al. This is an open access article distributed under the Creative Commons Attribution License, which permits unrestricted use, distribution, and reproduction in any medium, provided the original work is properly cited.

\begin{abstract}
The present paper provides general dentists with an introduction to the clinical applications and significance of magnetic resonance angiography (MRA) in the oral and maxillofacial regions. Specifically, the method and characteristics of MRA are first explained using the relevant MR sequences. Next, clinical applications to the oral and maxillofacial regions, such as identification of hemangiomas and surrounding vessels by MRA, are discussed. Moreover, the clinical significance of MRA for other regions is presented to elucidate future clinical applications of MRA in the oral and maxillofacial regions.
\end{abstract}

\section{Introduction}

It is very important and significant for doctors to determine the status of vascular-related diseases such as thrombosis and arteriosclerosis and to precisely visualize the threedimensional (3D) structure of the vasculature before surgery [1-3]. In dental fields, understanding the 3D structure of the vasculature is necessary and important for surgical operations on tumors and cysts. The most precise evaluation technique is conventional angiography (CR) using contrast medium. In CR, if the treatment should be applied for the status of vessels, the technique was continuously followed by the interventional radiology such as the embolization of arteries with arteriosclerosis. Therefore, the CR is a firm position for elucidating the status of vascular-related disease such as thrombosis and arteriosclerosis, and precisely visualization of $3 \mathrm{D}$ vasculatures at the preoperations. However, the risk of a new focal neurological deficit caused by $\mathrm{CR}$ is about $0.14 \% \sim 0.5 \%$, and this risk rises to $1.3 \% \sim 2.6 \%$ in patients investigated for stroke and transient ischemic attacks [4]. Therefore, it is important to identify the 3D structure of the vasculature of the arterial and venous systems as noninvasively as possible. To determine the $3 \mathrm{D}$ structure of the vasculature as noninvasively as possible, ultrasonography (US) is easy to use clinically. However, US cannot objectively visualize a wide area. Furthermore, the 
3D structure of the vasculature cannot be visualized using computed tomography (CT) without contrast medium. Of course, CT angiography (CTA) using contrast medium was a very useful tool for the $3 \mathrm{D}$ structure of the vasculature. However, the risk of complications in CTA should be higher because of iodine contrast medium.

Recent advances in magnetic resonance imaging (MRI), 3D data acquisition, and postprocessing technologies are expanding the potential applications of 3D displays [5-16]. As a noninvasive technique, magnetic resonance angiography (MRA) has been developed to identify and characterize peripheral blood vessels located in the thoracic and abdominal regions and the lower extremity [17-22]. Of course, MRA is very useful to visualize the $3 \mathrm{D}$ structure of the vasculature in the oral and maxillofacial regions, and it is clinically significant for evaluating the relationship between lesions and vessels in the oral region [14, 16, 23-26]. General dentists should know and easily understand the clinical applications of MRA in the oral and maxillofacial regions.

Therefore, the present paper provides a clear and easily understandable explanation of MRA, the method, and its characteristics, as well as its clinical applications and characteristics, for general dentists.

\section{Explanation of MRA, Its Method, and Characteristics Using the Relevant MR Sequences}

MRA commonly uses a bright-blood method, in which the signal from the moving protons is accentuated relative to the stationary protons through pulse sequences and measurement parameters. In fact, MRA is a technique using vessel flow or contrast agents such as gadolinium to detect vessel systems such as arteries and veins. Therefore, MRA has the least complication and is the easiest technique for $3 \mathrm{D}$ visualization of vessels, unlike CR using contrast medium such as iodine-related medium. At the same time, this technique is very useful for identifying vascular diseases and abnormalities, as well as the relationships between diseases and surrounding vessels.

The various techniques of MRA include MR sequences such as time of flight (TOF), phase contrast (PC), fresh blood imaging (FBI), and contrast-enhanced MRA [5-11, $13,15,16,27-37]$. MRA using TOF (TOF-MRA) is the most time-efficient method for obtaining MRA images. A single measurement is performed, with the stationary tissue signal suppressed relative to the flowing tissue signal [38]. MRA using PC (PC-MRA) is a technique in which the background tissue signal is subtracted from the flowenhanced image to produce flow-only images, analogous to digital subtraction X-ray angiography [38]. MRA as a new technique using FBI that has been applied to the $3 \mathrm{D}$ evaluation of vasculature [8-11, 13, 15, 16, 34-37]. FBI, which uses $3 \mathrm{D}$ half-Fourier fast advanced spin echo (FASE)triggered MR with electrocardiographic (ECG) gating, is one of the newest non-enhanced-MRA techniques [8-11, 13, 15, 16, 34-37]. Contrast-enhanced MRA makes good use of the contrast agent, which affects the relaxation times of the water protons in the nearby tissue. In MRA with contrast medium, as disadvantages, intravenous injection should be applied such as CR, and gadolinium could induce many kinds of complications including nephrogenic fibrosing dermopathy. MRA without contrast medium has no complications and no need of intravenous injections, and its technique can easily and additionally apply for many patients in whom vascular information should be evaluated. In the present paper, the focus is on three techniques (TOF, PC, and particularly FBI) as the main MRA techniques without contrast medium that are useful in the oral and maxillofacial regions.

\section{Clinical Applications of MRA for Lesions, Mainly Hemangiomas, in the Oral and Maxillofacial Regions}

On TOF- or PC-MRA, arteries and veins which are to some degree slow appear as bright homogeneous linear structures and stationary tissues except vessels with flow which appear for the most part blurred(Figure 1). Maximum intensity projection and multiplanar reconstruction techniques are used to visualize MRA data. Therefore, vessels with flow can be primarily visualized on MRA with these two techniques (Figure 1). On the other hand, vessels without flow cannot be visualized at all using MRA with TOF and PC techniques.

Of the two techniques in use for patients, PC-MRA is better for demonstrating slow-flowing blood vessels $(<10 \mathrm{~cm} / \mathrm{s})$, owing primarily to an optimized velocity-encoding gradient (VENC) and perhaps also to smaller saturation effects and better background suppression [39]. This sequence offers a better examination of the external carotid system than TOFMRA [39]. Though the main carotid artery and its branches can be visualized on PC-MRA, TOF-MRA can visualize the main carotid artery, but not its branches (Figure 1) [14]. In addition, 3D-PC-MRA has a better signal-to-noise ratio and a better spatial resolution than 2D-PC-MRA (Figure 2) [40]. Therefore, in the oral and maxillofacial regions, 3DPC-MRA routinely tends to be selected for additional and preoperative vascular evaluations in MR examinations.

However, in the oral and maxillofacial regions, the vasculature is complex, with blood flow rates varying from high in the carotid arteries to low in the lingual arteries. Thus, 3D visualization of the vasculature in these regions is very difficult to obtain even using 3D-PC-MRA. Therefore, in very recent reports, MRA using FBI (FBI-MRA) was used for the $3 \mathrm{D}$ evaluation of the vasculature in the oral and maxillofacial regions $[10,16,23]$. Most prior studies using FBI primarily examined the main vessels of the chest, abdomen, and lower extremities [9, 11, 13, 15, 34-37].

In previous reports, 3D-FBI-MRA could provide better $3 \mathrm{D}$ visualization of the vasculature of thin blood vessels, including the lingual and facial arteries, than 3D-PC-MRA (Figure 3) [16]. The axes of the lingual and facial arteries are under 2-3 mm. 3D-FBI-MRA permits the depiction of slow-flowing blood vessels by acquiring the images with ECG triggering during the slow-flow cardiac phase [31]. 3D-FBI-MRA can depict slow-flowing vessels due to the T2 effects, so that even relatively static blood vessels can 


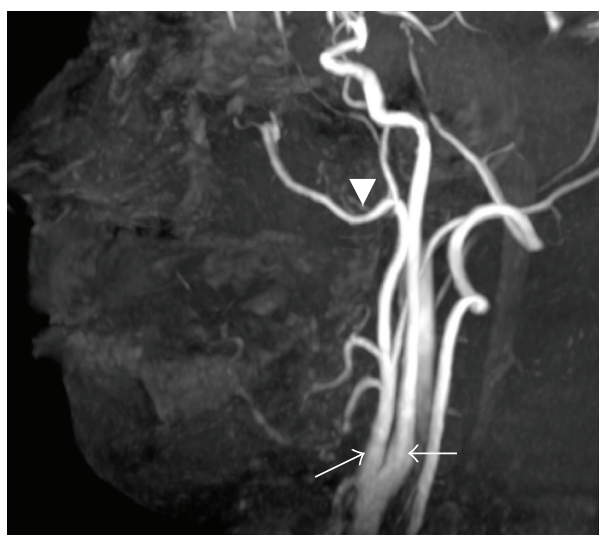

(a)



(b)

Figure 1: MRA using 3D-TOF (a) and 3D-PC (b) in the oral and maxillofacial regions of a healthy 33-year-old volunteer. (a) On MRA using 3D-TOF, mainly the carotid, maxillary, and facial arteries are shown clearly, but not its branches. The external carotid artery (arrow), internal carotid artery (black arrow), maxillary artery (arrowhead), and facial artery (black arrowhead). Acquisition time was 6 minutes and 39 seconds. (b) On MRA using 3D-PC, the carotid, maxillary, facial, and other peripheral arteries are shown in the same subject as in Figure 1(a). The external carotid artery (arrow), internal carotid artery (black arrow), maxillary artery (arrowhead), and facial artery (black arrowhead). Acquisition time was 10 minutes and 15 seconds.



(a)

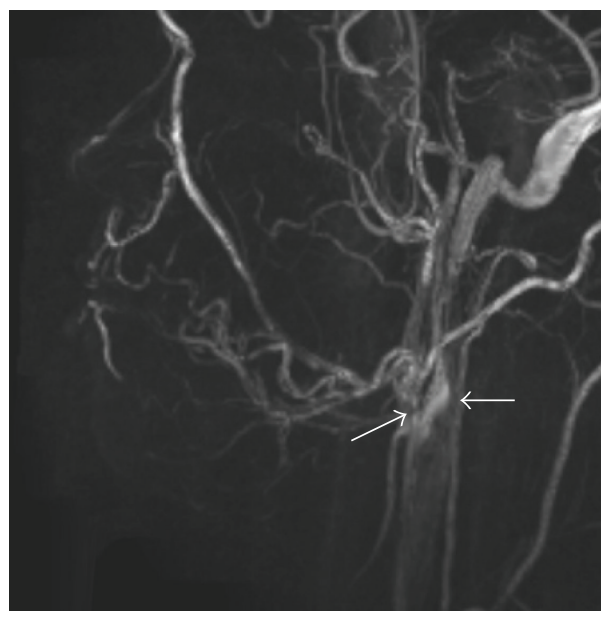

(b)

FIgURE 2: MRA using 2D-PC (a) and 3D-PC (b) in the oral and maxillofacial regions of a healthy 28-year-old volunteer. Three-dimensional (3D) PC-MRA has a better signal-to-noise ratio (SNR) and a better spatial resolution than 2D PC-MRA. (a) On MRA using 2D-PC, mainly the external (arrow) and internal carotid arteries (black arrow) and their branches are shown, but the image quality is low. Acquisition time was 9 minutes and 36 seconds. (b) On MRA using 3D-PC, the external (arrow) and internal carotid (black arrow) and many other branches are seen. The images are higher in quality in the same subject as in Figure 2(a). Acquisition time was 10 minutes and 15 seconds.

be depicted on images, unlike imaging with 3D-PC-MRA [31]. Therefore, 3D-FBI-MRA can even visualize vessels with very slow flow that cannot be visualized on 3D-PC-MRA $[7,9,13,16]$. In addition, the depiction level of thick blood vessels such as external and internal carotid arteries using 3D-FBI is the same as that using 3D-PC-MRA. In other word, peripheral small arteries look well visualized on 3D-FBI-MRA compared to 3D-PC-MRA, but not for large arteries. Carotid, maxillary, facial, and lingual arteries can be more precisely visualized on 3D-FBI-MRA without contrast medium than on 3D-PC-MRA (Figure 3). At the same time, the internal jugular, maxillary, facial, and lingual veins can also be better visualized on 3D-FBI-MRA without contrast medium than on 3D-PC-MRA (Figure 3).

3D-TOF- or PC-MRA depicts only blood vessels, but not mass lesions such as hemangiomas (Figure 4) [16, 23]. Therefore, the $3 \mathrm{D}$ relationship between the mass lesions such as hemangiomas and surrounding blood vessels should be visualized by fusing fat-saturation T2-weighted images with 3DPC-MRA [14]. By doing so, hemangiomas and surrounding 


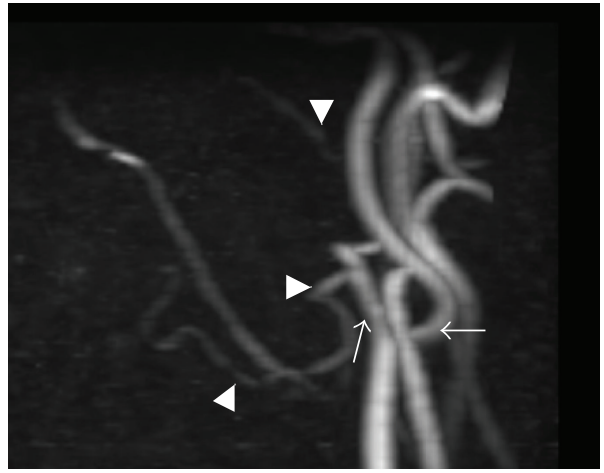

(a)

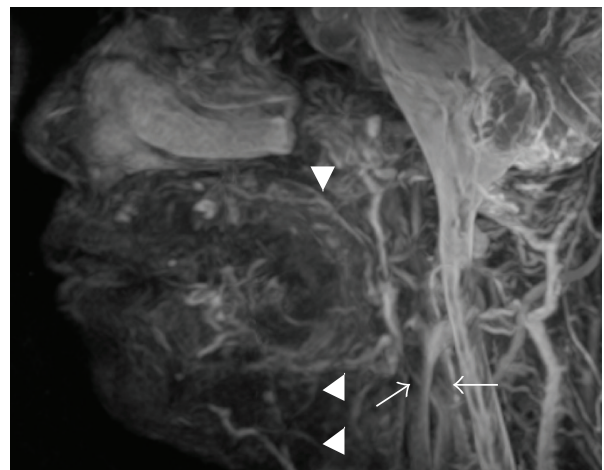

(b)

FIGURE 3: MRA using 3D-PC (a) and 3D-FBI (b) in the oral and maxillofacial regions of a healthy 48-year-old volunteer. MRA using 3D-FBI provides better 3D visualization of the vasculature of thin blood vessels, including the lingual and facial arteries, than 3D-PC-MRA. (a) On MRA using 3D-PC, the external (arrow) and internal carotid (black arrow), maxillary (arrowhead), and facial arteries (black arrowhead) are shown. Acquisition time was 10 minutes and 15 seconds. (b) On MRA using 3D-FBI, the external (arrow) and internal carotid (black arrow) and other peripheral vessels are shown. The maxillary (arrowhead), facial (black arrowhead), lingual (gray arrowhead), and further peripheral arteries are more precisely shown than in Figure 3(a) in the same subject. Acquisition time was 4 minutes and 5 seconds.

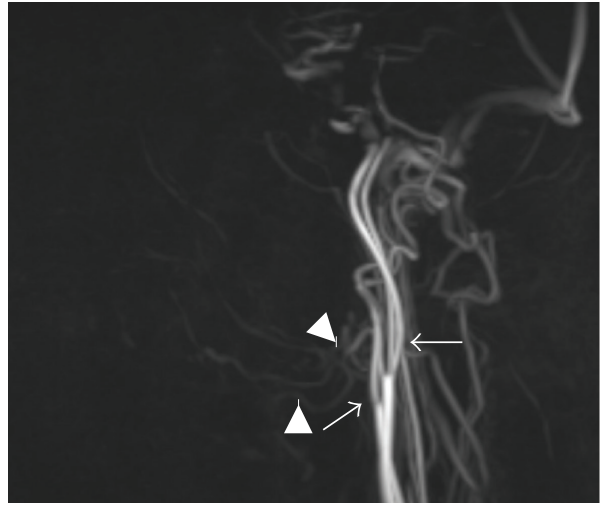

(a)

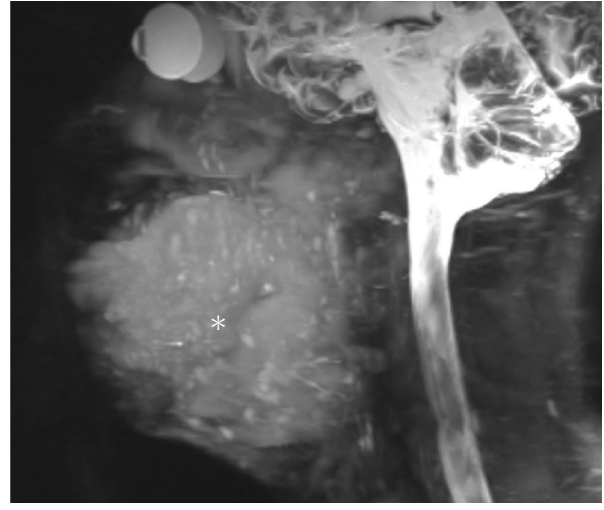

(b)

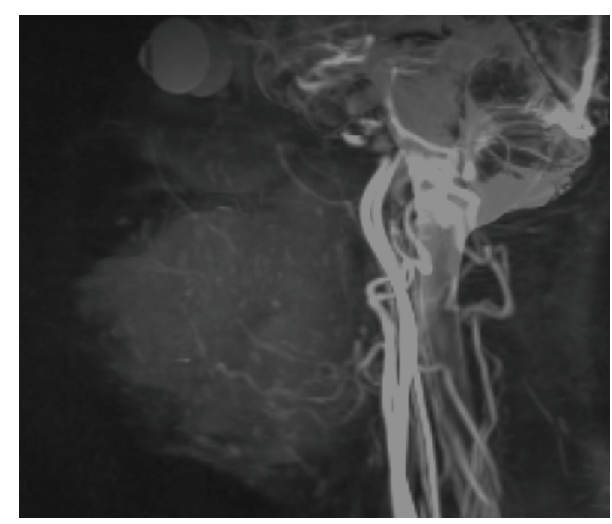

(c)

FIgURE 4: The production of a superimposed view of MRA using 3D-PC and a T2-weighted image in a 46-year-old patient with multiple hemangiomas of the bilateral tongue, lower lip, cheek, oral floor, masticator space, and right parotid space. (a) On MRA using 3D-PC, the external (arrow) and internal carotid arteries (black arrow), and the facial (arrowhead) and lingual arteries (black arrowheads) are shown, but the hemangiomas in the orofacial area are not seen. Acquisition time was 10 minutes and 15 seconds. (b) Fat-saturation T2-weighted image using 3D-FASE sequences of the head and neck. A hemangioma is visualized on this image (star). Acquisition time was 5 minutes and 8 seconds. (c) On fusing fat-saturation T2-weighted images with MRA by 3D-PC, hemangiomas and surrounding vessels can be visualized. However, image resolution of the fusion image is degraded compared to that of MRA using 3D-PC. 


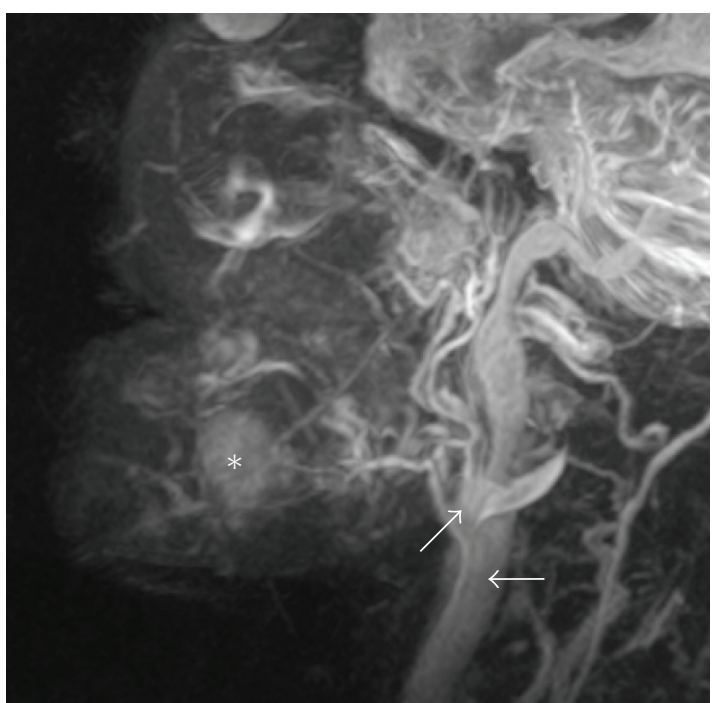

FIGURE 5: MRA by 3D-FBI in a 79-year-old patient with hemangiomas of the left oral floor. Both hemangiomas and thin blood vessels around tumor can be clearly visualized on MRA with 3D-FBI. The carotid arteries (arrow), internal jugular vein (black arrow), lingual artery, lingual vein, facial artery, facial vein, maxillary artery, and maxillary vein are identified as bright homogeneous linear structures. At the same time, hemangiomas (star) are clearly visualized as the mass with a high signal intensity. Acquisition time was 3 minutes and 28 seconds.

vessels can be visualized on the fusion images (Figure 4). The software to allow superimposition of both 3D-PC-MRA and T2-weighted images is integral to the MR system. However, image resolution degradation is a disadvantage of fusion imaging (Figure 4). Deterioration of the contrast-to-noise and signal-to-noise ratios on superimposed views occurs because noise on the respective images is doubled without increasing the signal intensity of the tumor and vessels [14]. In addition, production of the superimposed view might be unsuccessful if the patient should move in the time gap between capture of the respective images [14].

On the other hand, both hemangiomas and thin blood vessels around tumors can be clearly visualized on 3DFBI-MRA without fusion of MRA with tumor images (Figure 5) [23]. The carotid arteries, internal jugular veins, lingual arteries, lingual veins, facial arteries, facial veins, maxillary arteries, and maxillary veins can be seen as bright homogeneous linear structures (Figure 5). At the same time, hemangiomas are clearly visualized as masses with high signal intensity (Figure 5). Except for the carotid arteries, 3D-FBI-MRA is better than 3D-PC-MRA based on visual scores despite the short acquisition time [23]. Artifacts from patient movement and shortened acquisition times reduce image quality more with 3D-PC-MRA than with 3D-FBI.

Another advantage of 3D-FBI-MRA is that one can distinguish between arteries and veins without the use of contrast medium [8-11, 13, 15, 16, 23, 34-37]. The blood vessels depicted on 3D-FBI-MRA are most of the arteries, including the carotid, maxillary, facial, lingual, and other arteries (Figure 6). Flow-spoiled gradient pulses do not affect the signal intensity of stationary background tissues. Veins are similarly less affected by the flow-spoiled pulse during diastole and systole as a result of their relatively constant slow flow throughout the cardiac cycle [15]. By applying the flow-spoiled pulses, the signal intensity difference between diastole and systole in the arteries is increased. Thus, diastolic and systolic subtraction provides better delineation of the arteries. However, except for 3D-FBI, the distinction between arteries and veins has not been possible with MRA sequences such as 3D-TOF and 3D-PC without contrast medium so far. It is hoped that 3D-FBI-MRA will be a significant, epochmaking technique.

However, there are some potential disadvantages with 3D-FBI-MRA for clinical applications in the oral and maxillofacial regions $[16,34-37]$. The first is that the selection of the appropriate flow-spoiled gradient is important for determining the image quality of hemangiomas and surrounding vessels. Even a slight mistake affects the visualization of branch arteries, such as the maxillary and lingual arteries [16, 23]. To select the appropriate flow-spoiled gradient pulses is very difficult for operators, because blood vessels in the oral and maxillofacial regions have a wide range of flow rates, from high to static. The second disadvantage is that 3D-FBIMRA is sensitive to movement. Therefore, the images tend to be degraded in patients with movements of the mandible and tongue. Moreover, the third disadvantage is that patients with arrhythmias or tachycardias are not suitable candidates for FBI examinations, because ECG triggering is used.

\section{Future Uses of MRA for Lesions in the Oral and Maxillofacial Regions}

MRA cannot perfectly replace CR for examinations of vascular-related diseases and suspected hemangiomas in the oral and maxillofacial regions. That is why MRA cannot have more contrast between vessels and tissues except vessels and cannot be more precisely visualized than CR. However, performing MRA for diseases in the oral and maxillofacial regions should be recommended in addition to common MR examinations before CR. In particular, 3D-FBI-MRA is relatively easy to perform and can precisely visualize thin vessels except a little difficulty of parameters setting. Its technique can distinguish between arteries and veins without the use of contrast medium. Therefore, examinations using 3D-FBI-MRA are very useful and important for preoperative evaluations for various kinds of diseases, in particular the 3D relationship between the vasculature and diseases. MRA with contrast medium is also important. At present, higher spatial resolution gadolinium-MRA can be achieved at 3 Tesla with a sustained or greater signal-to-noise ratio of enhanced vasculature, relative to 1.5 Tesla [41]. Therefore, MRA with higher resolution in the oral and maxillofacial regions can be achieved with a 3-Tesla MR system. Very recently, it has been found that existing MRA is suboptimal for assessing the hemodynamics of arteriovenous malformations [42-50]. As a completely noninvasive method, 4D dynamic MRA offers hemodynamic information with a temporal resolution of 50$100 \mathrm{~ms}$ for the evaluation of arteriovenous malformations 




(a)

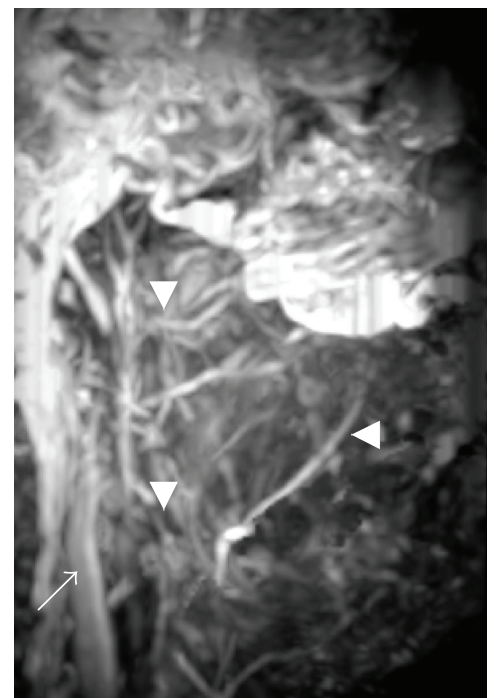

(b)

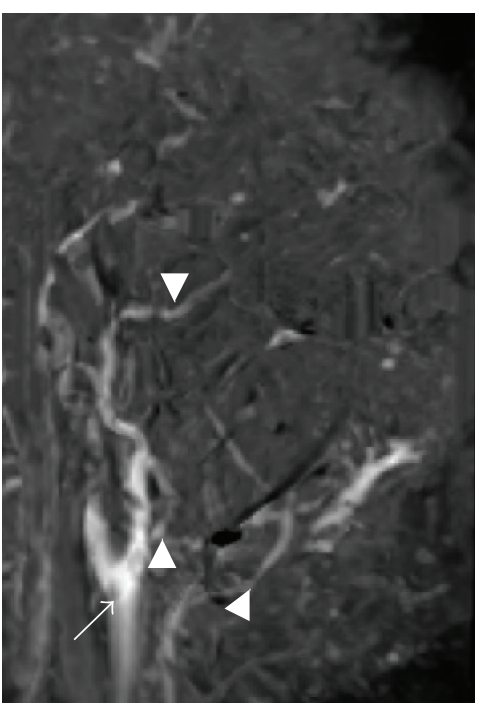

(c)

Figure 6: MRA by 3D-FBI in the oral and maxillofacial regions of a 49-year-old healthy volunteer. MRA using 3D-FBI can distinguish between arteries (a) and veins (b) without the use of contrast medium. (a) Images acquired during diastole. The carotid artery, internal jugular vein, maxillary artery, maxillary vein, facial artery, facial vein, lingual artery, and lingual vein are visualized. Acquisition time was 3 minutes and 36 seconds. (b) Images acquired during systole. The internal jugular vein (arrow), maxillary vein (arrowhead), facial vein (black arrowhead), and lingual vein (gray arrowhead) are depicted. Acquisition time was 3 minutes and 36 seconds. (c) The subtraction image of the systolic image (b) from the diastolic image (a) that depicts the carotid (arrow), maxillary (arrowhead), facial (black arrowhead), and lingual arteries (gray arrowhead).

and can complement existing methods, such as dynamic subtraction analysis and TOF MRA [42-46]. Therefore, the evaluation of hemodynamics in hemangiomas will be performed by MRA in the oral and maxillofacial regions in the future.

\section{Conflict of Interests}

No potential conflict of interests were disclosed.

\section{Acknowledgment}

This study was supported in part by grants-in-aid for scientific research from the Ministry of Education, Culture, Sports, Science and Technology of Japan and from Kitakyushu city to Y. Maki.

\section{References}

[1] J. Bian, L. Sha, C. Yang, and C. S. Sun, “Three-dimensional dynamic contrast-enhanced MR angiography for evaluating recipient vessels in orthotopic liver transplantation," Hepatobiliary and Pancreatic Diseases International, vol. 7, no. 5, pp. 476-480, 2008.

[2] K. D. Hagspiel, K. Nandalur, T. L. Pruett et al., "Evaluation of vascular complications of pancreas transplantation with high-spatial-resolution contrast-enhanced MR angiography," Radiology, vol. 242, no. 2, pp. 590-599, 2007.

[3] N. Hidajat, H. Stobbe, V. Griesshaber, R. J. Schroder, and R. Felix, "Portal vein thrombosis: etiology, diagnostic strategy, therapy and management," Vasa-Journal of Vascular Diseases, vol. 34, no. 2, pp. 81-92, 2005.

[4] J. E. Heiserman, B. L. Dean, J. A. Hodak et al., "Interventional neurovascular disease: avoidance and management of complications and review of the current literature," Journal of Neurosurgical Sciences, vol. 55, no. 3, pp. 233-242, 2011.

[5] S. Hähnel, M. Hartmann, O. Jansen, and K. Sartor, "Persistent hypoglossal artery: MRI, MRA and digital subtraction angiography," Neuroradiology, vol. 43, no. 9, pp. 767-769, 2001.

[6] P. P. Kesava and P. A. Turski, "Magnetic resonance angiography of vascular malformations," Neuroimaging Clinics of North America, vol. 8, no. 2, pp. 349-370, 1998.

[7] A. Tetsumura, N. Yoshino, I. Yamada, and T. Sasaki, "Head and neck haemangiomas: contrast-enhanced three-dimensional MR angiography," Neuroradiology, vol. 41, no. 2, pp. 140-143, 1999.

[8] M. Miyazaki, F. Tateishi, S. Sugiura, Y. Machida, Y. Kassai, and H. Abe, "Fresh blood imaging at 0.5-T: natural blood contrast 3D MRA within single breathhold," in Proceedings of the 6th Annual Meeting of International Society for Magnetic Resonance in Medicine, p. 692, Denver, Colo, USA, 2000.

[9] M. Miyazaki, S. Sugiura, F. Tateishi, H. Wada, Y. Kassai, and H. Abe, "Non-contrast-enhanced MR angiography using 3D ECG-synchronized half-Fourier fast spin echo," Journal of Magnetic Resonance Imaging, vol. 12, no. 5, pp. 776-783, 2000.

[10] T. Kogure, K. Kogure, M. Iizuka, A. Ino, and M. Ishii, "Effective use of flow-spoiled FBI and time-SLIP methods in the diagnostic study of an aberrant vessel of the head and neck: 'left jugular venous steal by the right jugular vein," Journal of Magnetic Resonance Imaging, vol. 32, no. 2, pp. 429-433, 2010.

[11] A. Ono, K. Murase, T. Taniguchi et al., "Deep vein thrombosis using noncontrast-enhanced MR venography with electrocardiographically gated three-dimensional half-fourier FSE: 
preliminary experience," Magnetic Resonance in Medicine, vol. 61, no. 4, pp. 907-917, 2009.

[12] Y. Machida, H. Kanazawa, Y. Kassai, H. Takai, S. Sugiura, and M. Miyazaki, "Effects of gradient moment nulling in 3D half-Fourier FSE. bright blood imaging," in Proceedings of the 8th Meeting of the International Society for Magnetic Resonance in Medicine, p. 1829, International Society for Magnetic Resonance in Medicine, Berkeley, Calif, USA, 2000.

[13] M. Miyazaki, H. Takai, S. Sugiura, H. Wada, R. Kuwahara, and J. Urata, "Peripheral MR angiography: separation of arteries from veins with flow-spoiled gradient pulses in electrocardiography-triggered three-dimensional half-Fourier fast spin-echo imaging," Radiology, vol. 227, no. 3, pp. 890896, 2003.

[14] T. Tanaka, Y. Morimoto, H. Takano et al., “Three-dimensional identification of hemangiomas and feeding arteries in the head and neck region using combined phase-contrast MR angiography and fast asymmetric spin-echo sequences," Oral Surgery, Oral Medicine, Oral Pathology, Oral Radiology and Endodontology, vol. 100, no. 5, pp. 609-613, 2005.

[15] J. Urata, M. Miyazaki, H. Wada, T. Nakaura, Y. Yamashita, and M. Takahashi, "Clinical evaluation of aortic diseases using nonenhanced MRA with ECG-triggered 3D half-fourier FSE," Journal of Magnetic Resonance Imaging, vol. 14, no. 2, pp. 113$119,2001$.

[16] T. Tanaka, M. Oda, S. Kito et al., "Noninvasive identification of peripheral vessels of oral and maxillofacial regions using electrocardiography-triggered three-dimensional fast asymmetric spin-echo sequences," Oral Medicine, Oral Pathology, Oral Radiology, and Endodontology, vol. 112, no. 4, pp. 493501, 2011.

[17] F. von Ziegler and M. A. Costa, "The role of CT and MRI in the assessment of peripheral vascular disease," Current Cardiology Reports, vol. 9, no. 5, pp. 412-419, 2007.

[18] G. Rigatelli, "Renovascular disease imaging: today more than ever the invasive cardiologist may make the difference," International Journal of Cardiology, vol. 113, no. 2, pp. 149$152,2006$.

[19] J. P. Earls and S. C. Shaves, "MR angiography of the thoracic, abdominal, and extremity venous system," Magnetic Resonance Imaging Clinics of North America, vol. 6, no. 2, pp. 417-435, 1998.

[20] J. P. Carpenter, R. S. Owen, G. A. Holland et al., "Magnetic resonance angiography of the aorta, iliac, and femoral arteries," Surgery, vol. 116, no. 1, pp. 17-23, 1994.

[21] D. Chan, M. E. Anderson, and B. L. Dolmatch, "Imaging evaluation of lower extremity infrainguinal disease: role of the noninvasive vascular laboratory, computed tomography angiography, and magnetic resonance angiography," Techniques in Vascular and Interventional Radiology, vol. 13, no. 1, pp. 11-22, 2010.

[22] H. Ersoy and F. J. Rybicki, "MR angiography of the lower extremities," American Journal of Roentgenology, vol. 190, no. 6, pp. 1675-1684, 2008.

[23] M. Oda, T. Tanaka, S. Kito et al., "Magnetic resonance angiography with fresh blood imaging for identification of hemangiomas and blood vessels around hemangiomas in oral and maxillofacial regions," Oral Surgery, Oral Medicine, Oral Pathology, and Oral Radiology, vol. 113, no. 4, pp. 559-566, 2012.

[24] K. Zhi, W. Ren, H. Zhou, Y. Wen, and Y. Zhang, "Management of parapharyngeal-space tumors," Journal of Oral and Maxillofacial Surgery, vol. 67, no. 6, pp. 1239-1244, 2009.
[25] L. Goffinet, B. Laure, T. Tayeb et al., "An arteriovenous fistula of the maxillary artery as a complication of Le Fort I osteotomy," Journal of Cranio-Maxillofacial Surgery, vol. 38, no. 4, pp. 251-254, 2010.

[26] M. Loukas, C. R. Kinsella Jr., T. Kapos, R. S. Tubbs, and S. Ramachandra, "Anatomical variation in arterial supply of the mandible with special regard to implant placement," International Journal of Oral and Maxillofacial Surgery, vol. 37, no. 4, pp. 367-371, 2008.

[27] F. Aspestrand and A. Kolbenstvedt, "Vascular mass lesions and hypervascular tumors in the head and neck characteristics at CT, MR imaging and angiography," Acta Radiologica, vol. 36, no. 2, pp. 136-141, 1995.

[28] M. Crecco, R. Floris, A. Vidiri et al., "Venous angiomas: plain and contrast-enhanced MRI and MR angiography," Neuroradiology, vol. 37, no. 1, pp. 20-24, 1995.

[29] M. Oelerich, M. G. Lentschig, P. Zunker, P. Reimer, E. J. Rummeny, and G. Schuierer, "Intracranial vascular stenosis and occlusion: comparison of 3D time-of-flight and 3D phasecontrast MR angiography," Neuroradiology, vol. 40, no. 9, pp. 567-573, 1998.

[30] J. R. Pernicone, K. E. Thorp, M. V. Ouimette, J. E. Siebert, and E. J. Potchen, "Magnetic resonance angiography in intracranial vascular disease," Seminars in Ultrasound CT and MRI, vol. 13, no. 4, pp. 256-273, 1992.

[31] A. Uchino, K. Hasuo, S. Matsumoto, A. Mizushima, and K. Masuda, "Postcontrast 3D-TOF-MR angiography of intracranial venous angiomas," Radiation Medicine, vol. 13, no. 4, pp. 157-161, 1995.

[32] M. Goyen and L. J. G. Heuser, "Improved peripheral MRA using multi-velocity-encoding phase contrast-enahanced MRA techniques," Acta Radiologica, vol. 41, no. 2, pp. 139-141, 2000.

[33] P. Reimer and M. Boos, "Phase-contrast MR angiography of peripheral arteries: technique and clinical application," European Radiology, vol. 9, no. 1, pp. 122-127, 1999.

[34] E. T. D. Hoey, A. Ganeshan, R. Puni, J. Henderson, and P. M. Crowe, "Fresh blood imaging of the peripheral vasculature: an emerging unenhanced MR technique," American Journal of Roentgenology, vol. 195, no. 6, pp. 1444-1448, 2010.

[35] P. Wong, M. J. Graves, and D. J. Lomas, "Interactive twodimensional fresh blood imaging: a feasibility study," European Radiology, vol. 19, no. 4, pp. 904-911, 2009.

[36] P. Storey, I. P. Atanasova, R. P. Lim et al., "Tailoring the flow sensitivity of fast spin-echo sequences for noncontrast peripheral MR angiography," Magnetic Resonance in Medicine, vol. 64, no. 4, pp. 1098-1108, 2010.

[37] R. P. Lim, E. M. Hecht, J. Xu et al., "3D nongadoliniumenhanced ECG-gated MRA of the distal lower extremities: preliminary clinical experience," Journal of Magnetic Resonance Imaging, vol. 28, no. 1, pp. 181-189, 2008.

[38] M. A. Brown and R. C. Semelka, MRI Basic Principles and Applications, Wiley-Liss, New York, NY, USA, 2nd edition, 1999.

[39] J. Huston III, D. A. Rufenacht, R. L. Ehman, and D. O. Wiebers, "Intracranial aneurysms and vascular malformations: comparison of time-of- flight and phase-contrast MR angiography," Radiology, vol. 181, no. 3, pp. 721-730, 1991.

[40] S. C. Thomas, E. D. James, and C. M. Robert, Christensen's Physics of Diagnostic Radiology, Lea \& Febiger, philadelphia, $\mathrm{Pa}$, USA, 4th edition, 1990.

[41] F. J. Londy, S. Lowe, P. D. Stein et al., "Comparison of 1.5 and 3. $0 \mathrm{~T}$ for contrast-enhanced pulmonary magnetic resonance 
angiography," Clinical and Applied Thrombosis/Hemostasis, vol. 18, no. 2, pp. 134-139, 2012.

[42] S. Yu, L. Yan, Y. Yao et al., "Noncontrast dynamic MRA in intracranial arteriovenous malformation (AVM): comparison with time of flight (TOF) and digital subtraction angiography (DSA)," Magnetic Resonance Imaging, vol. 30, no. 2, pp. 869877, 2012.

[43] D. R. Hadizadeh, G. M. Kukuk, D. T. Steck et al., "Noninvasive evaluation of cerebral arteriovenous malformations by $4 \mathrm{D}$ MRA for preoperative planning and postoperative followup in 56 patients: comparison with DSA and intraoperative findings," American Journal of Neuroradiology, vol. 33, no. 6, pp. 1095-1101, 2012.

[44] U. Kramer, U. Ernemann, S. Mangold et al., "Diagnostic value of high spatial and temporal resolution time-resolved MR angiography in the workup of peripheral high-flow vascular malformations at 1.5 Tesla," International Journal of Cardiovascular Imaging, vol. 28, no. 4, pp. 823-834, 2012.

[45] J. Pekkola and M. Kangasniemi, "Posterior fossa dural arteriovenous fistulas: diagnosis and follow-up with timeresolved imaging of contrast kinetics (TRICKS) at 1.5 T," Acta Radiologica, vol. 52, no. 4, pp. 442-447, 2011.

[46] L. Schuster, E. Schenk, F. Giesel et al., "Changes in AVM angio-architecture and hemodynamics after stereotactic radiosurgery assessed by dynamic MRA and phase contrast flow assessments: a prospective follow-up study," European Radiology, vol. 21, no. 6, pp. 1267-1276, 2011.

[47] L. Boussel, A. Cernicanu, L. Geerts et al., "4D time-resolved magnetic resonance angiography for noninvasive assessment of pulmonary arteriovenous malformations patency," Journal of Magnetic Resonance Imaging, vol. 32, no. 5, pp. 1110-1116, 2010.

[48] M. I. Vargas, D. Nguyen, M. Viallon et al., "Dynamic MR angiography (MRA) of spinal vascular diseases at 3 T," European Radiology, vol. 20, no. 10, pp. 2491-2495, 2010.

[49] D. R. Hadizadeh, J. Gieseke, G. Beck et al., "View-sharing in keyhole imaging: partially compressed central k-space acquisition in time-resolved MRA at 3. 0 T," European Journal of Radiology, vol. 80, no. 2, pp. 400-406, 2011.

[50] S. Nishimura, T. Hirai, A. Sasao et al., "Evaluation of dural arteriovenous fistulas with 4D contrast-enhanced MR angiography at 3 T," American Journal of Neuroradiology, vol. 31 , no. 1 , pp. $80-85,2010$. 


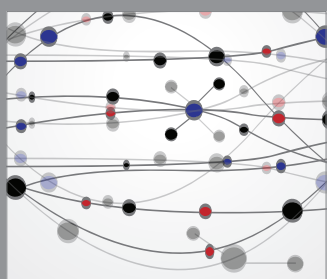

The Scientific World Journal
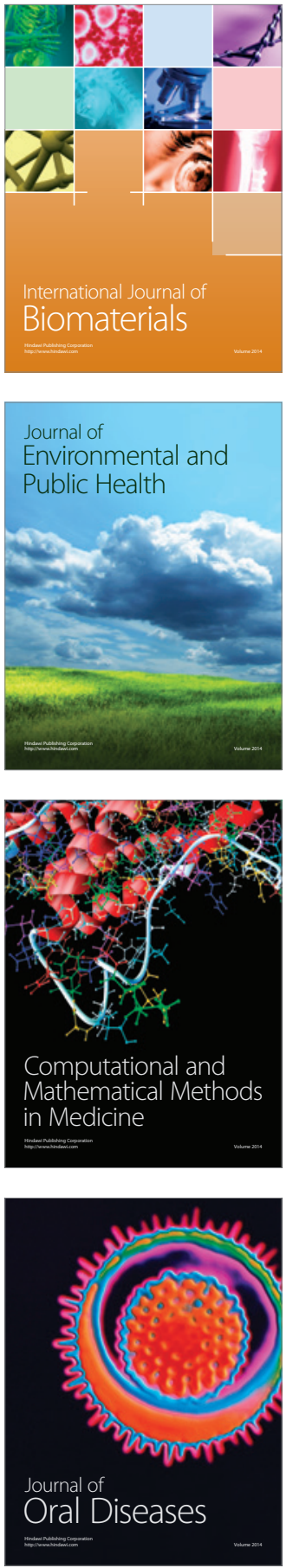
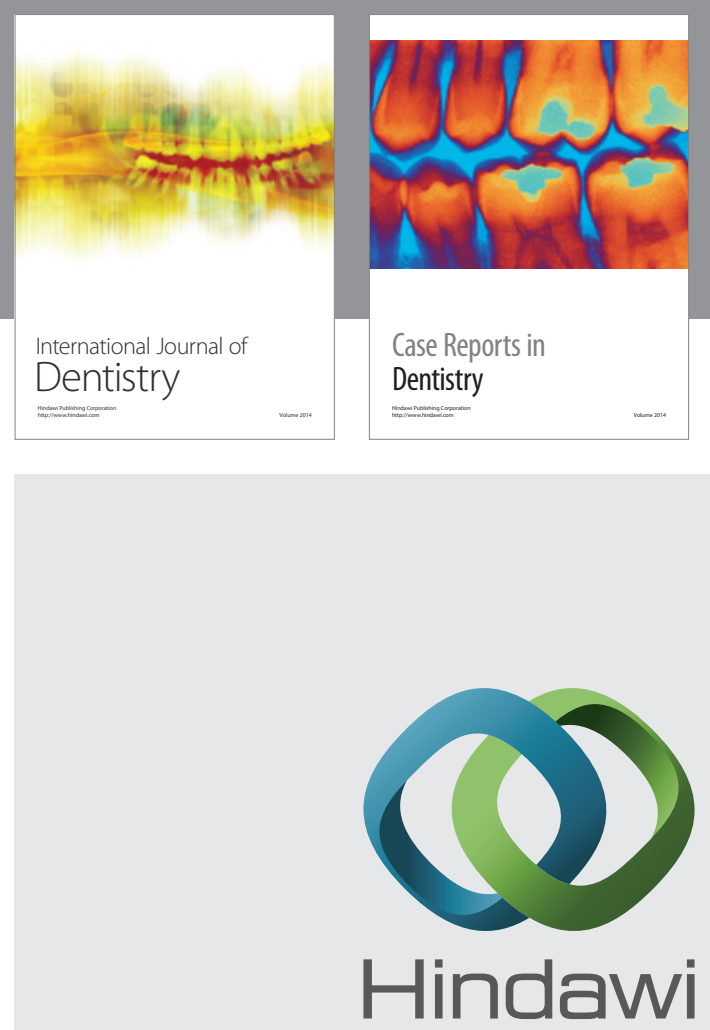

Submit your manuscripts at

http://www.hindawi.com
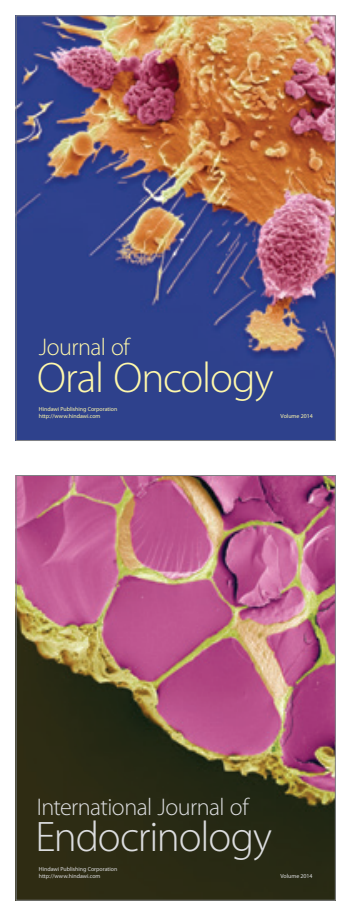
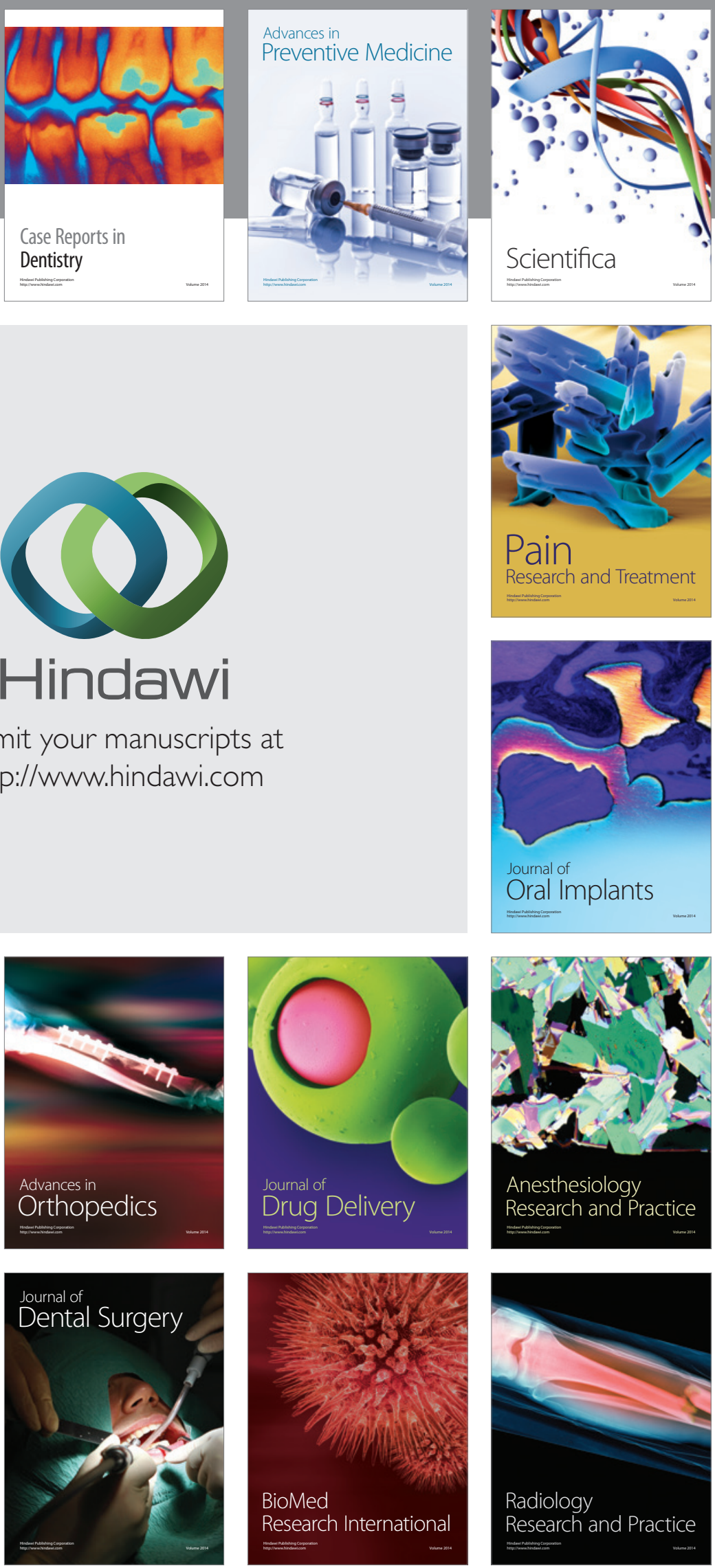